\title{
Optimal Offshore Wind Farms' Collector Design based on the Multiple Travelling Salesman Problem and Genetic Algorithm
}

\author{
Francisco M. Gonzalez-Longatt \\ Coventry University \\ Faculty of Computing and Engineering \\ Coventry, United Kingdom \\ fglongatt@ieee.org
}

\begin{abstract}
The capital cost of the electrical network of a large offshore wind farm constitutes a significant proportion of the total cost. Finding the optimal design of electrical network is imperative task and it is addressed in this paper. The objective of this paper is to present a methodology for the optimal design for the offshore wind farms' collector system; it is based on the Multiple Travelling Salesman Problem and Genetic Algorithm. A cost model has been developed that includes a more realistic treatment of the cost of step-up transformers and undersea cables. These improvements make this cost model more detailed than others that are currently in use. Optimization model is specifically designed for offshore wind farms' collector system and it considers different cable cross sections when designing the radial arrays. A novel optimization method is used; it is based on an improved Genetic Algorithm and includes a specific application of the Open-Multiple Traveling Salesmen Problem (fsomTSP) considering a special gene coding developed for this specific formulation. The proposed approach is tested with a hypothetical wind farm where the convergence is examined versus number of wind turbines.
\end{abstract}

Index Terms-- Electric distribution system, genetic algorithms, optimization methods, offshore wind farm, wind power generation.

\section{INTRODUCTION}

Offshore wind power is growing solidly, European countries installed and connected to the grid 293 offshore wind turbines - representing $1.165 \mathrm{GW}$ of capacity - in 2012, it represents a $33 \%$ increase over the $874 \mathrm{MW}$ installed in 2011 [1]. Offshore wind power generation is very attractive for several reasons [2]: (1) Offshore wind farms will not take up valuable land resources, (2) The use offshore locations will mean that the wind farm will have minimal landscape and visual impact and will not produce noise pollution, (3) Wind flow will not be perturbed by structures or forests, thereby the winds striking the turbine blades will be stronger and steadier causing an increase in the performance of the turbine, (4) Offshore wind turbine designs with larger single unit rated power than onshore designs are available, these larger ratings allow improved economies of scale and (5) Sea water affords an opportunity for accessible and low cost component cooling.

Large-scale offshore wind farms are being developed all around the world. A large off-shore wind farm may consist of hundreds of individual wind turbine (WT) generators connected to a medium voltage (MV) network, or collector system, that is itself connected to the transmission system at the point of interconnection (POI). The local WT collection system begins with the transformers that are installed at every wind turbine, usually in the base of the tower, it is called simply collector system. The MV distribution system will consist of one or more feeders and these feeders are connected to offshore transformer substations that collect the electricity produced by the WTs connected to the feeders. Offshore wind farms typically consist of several transformer substations that collect the electricity produced by the wind farm, an integration system is then used to transmit the energy from all of these individual substations to the central or terminal substation. Finally a high voltage transmission system is used to connect the terminal substation and the POI (see Fig. 1).

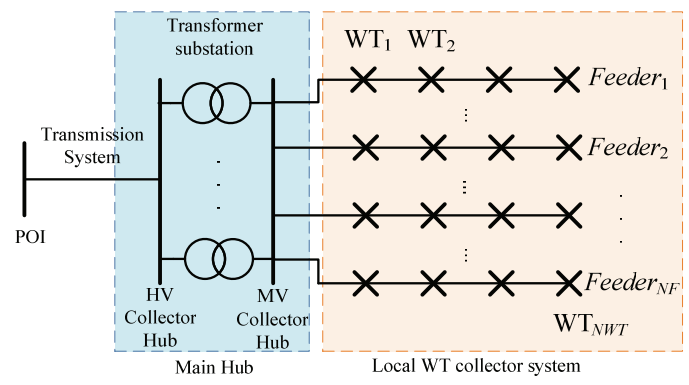

Fig. 1. Typical electrical network scheme for a wind farm.

The design of local WT collection (LWTC), integration and transmission systems for an offshore wind farm, including topology and component selection, should be carefully specified to strike the necessary balance between technical performance and economic cost [3]. Optimization of the 
design of the offshore distribution system therefore offers an opportunity to achieve a remarkable reduction in the total cost of an offshore wind farm. The number of variables involved in this problem and the optimal solution require the use of computational optimization. The search space for an optimal solution may therefore contain many sub-optimal points and as the problem dimensions increase, finding the global optimum becomes extremely difficult. The intelligentheuristic methods are much suitable to deal with such multivariable, highly nonlinear, optimization NP-hard problem.

There are several papers dealing with the optimization of the collector system on offshore wind farm. Optimization of electrical connection schemes for offshore wind farms considering solely investment cost models is resolved using GA in [4]. Nandingam et al [5] has used geometric programming to solve an optimization model based on cost, loss and reliability, for a single main substation and this approach has been tested using a small wind farm. The standard GA is improved with immune algorithm in [3] and the cost model of investment and operation are included; however, this approach could be improved by considering different cable cross sections in the design of local WT collection system and one special problem formulation specific for radial topology.

In this paper a novel approach to solving the problem of optimal electric network design for large offshore wind farms is presented. It is based on improved GA and includes a new approach based on a modified approach of the Travelling Salesman Problem (mTSP), it has been used for designing the radial arrays, and furthermore, the cost model has been improved by including more realistic terms. The optimization model and the solution algorithm proposed are shown in section two and three respectively. Section four presents the results of numerical experiments to show the capabilities and application of the approach for the optimal offshore wind farms' collector design.

\section{OPTIMIZARION MODEL}

The optimal design of the collector system supporting an offshore wind farm is dependent on the following: (i) the topology of the local WT collection system, (ii) satisfying any technical restrictions (voltage regulations, power losses, etc) and (iii) minimizing the total cost. In this section an optimization model which is suitable to find the optimum local WT collection system of an offshore wind farm is described. In this section a cost model for whole elements involved on the collector system is showed.

\section{A. Collector System Cost model}

The total investment cost $\left(C_{\text {total }}\right)$ is calculated assuming that the whole investment is made in the first year and paid of during the life time of the wind farm. In addition, it is assumed that some profit shall be made.
The annual investment cost of the collector system design of an offshore wind farm is calculated as the sum of the annual investment cost for the MV cable $\left(C_{C B}\right)$ and the cost of the transformer installed at each WT $\left(C_{W T T}\right)$ :

$$
\begin{gathered}
C_{\text {total }}=C_{C B}+C_{W T T} \\
=\frac{r(1+r)^{N_{L T}}}{(1+r)^{N_{L T}}} \frac{100}{100-P R} C_{i n v}=K C_{i n v}
\end{gathered}
$$

where $N_{L T}$ is the wind farm life time, $r$ is the interest rate, $P R$ is the profit in percent, and $C_{i n v}$ is the total investment cost necessary for the wind farm electrical system and $K$ is a constant.

The optimization problem can be described as:

$$
\begin{gathered}
\min \left[C_{\text {Total }}\right] \\
\min \left[K\left(\sum_{i=1}^{N_{F}} C_{F i}+C_{W T}\right)\right]
\end{gathered}
$$

where $N_{F}$ is the number of feeders on the substation, $C_{F i}$ is the total investment cost of the $i$ th feeder $\left(F_{i}\right)$, and $C_{W T}$ is the total investment cost of the step-up transformers connected to the WTs. Subject to:

$$
\begin{gathered}
I_{L m}<I_{\text {rated }}\left(c_{m}\right), \quad m \in F_{i} \\
\sum_{m \in X}\left|\Delta V_{m}\right|>\Delta V_{\min }, \quad m \in F_{i} \\
F_{i} \bigcap_{i, j \in \mathbf{X}} F_{j}=\varnothing
\end{gathered}
$$

where $I_{L m}$ is the nominal current in the $m$ th section of the $F_{i}$, and $I_{\text {rated }}\left(c_{m}\right)$ is the rated current for the $c_{m}$ type of conductor.

\section{1) Transformer connected to WT}

The cost cots model for the step up transformers at each wind turbine $\left(C_{W T T}\right)$, is described as:

$$
C_{W T T}=N_{W T} C_{W T}
$$

where $C_{W T}$ is cost of the transformer and $N_{W T}$ is the number of wind turbine in the wind farm.

\section{2) $\mathrm{MV}$ Cable}

The number of WT connected to one radial feeder of the collecting system depends on the maximum power that can be transmitted by the MW cable whilst satisfying certain power quality constraints. The necessary current carrying capacity in one radial configuration will vary between different sections of the feeder and thus using different cross sections of cable will reduce the cost without compromising performance.

Fig. 2 shows the configuration of the $i$ th feeder, each wind turbine injects current $\left(I_{W T m}\right)$ into the feeder, the cable size $c_{m}$ for each section $m$, carrying $I_{L m}$ current are depicted from the point of connection at the offshore transformer substation (PCC). The cable sizes $\left(c_{m}\right)$ are based on the criteria: the load 
current and the maximum voltage drop permissible $\left(\Delta V_{\text {min }}\right)$, considering the correspondent length in each section $\left(d_{m}\right)$.

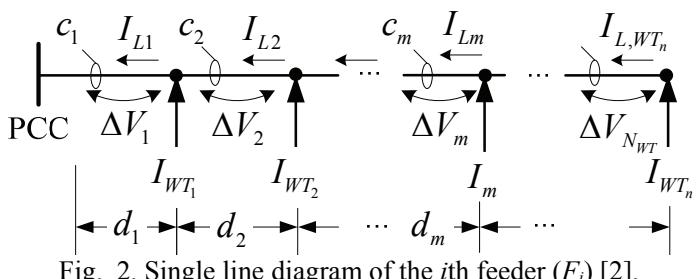

The total investment cost of the $i$ th feeder $\left(C_{F i}\right)$ on the offshore transformer substation can be obtained as:

$$
\mathrm{C}_{F i}=C S_{i}+C C_{i}
$$

where $C S_{i}$ is the shipping and installation cost for the undersea MW cables in the $i$ th feeder and $C C_{i}$ is the total cost of the MV cables:

$$
C C_{i}=\sum_{m \in F_{i}} C B\left(c_{m}\right) d_{m}
$$

where: $C B\left(c_{m}\right)$ is the cost of the $c_{m}$ type of conductor used in the section $m, d_{m}$ is the length of section $m$ the $i$ th feeder. The cost of the MV cable used in each section of the local WT collection system is a function of conductor area $A\left(c_{m}\right)$ and the rated voltage $\left(V_{\text {rated }}\right)[3]$ :

$$
\begin{gathered}
C B\left(c_{m}\right)=\alpha_{3}+\alpha_{4} e^{\frac{\beta_{2} S_{\text {ratad }}\left(c_{m}\right)}{10^{8}}} \\
S_{\text {rated }}\left(c_{m}\right)=\sqrt{3} V_{\text {rated }} I_{\text {rated }}\left(c_{m}\right)
\end{gathered}
$$

where: $I_{\text {rated }}\left(c_{m}\right)$ is the rated current for the $c_{m}$ type of conductor, $\alpha_{3}$ is an offset constant equal to $72845, \alpha_{4}$ is a slope constant equal to $1.0177 \times 10^{4}$ and the exponent $\beta_{2}$ is equal to $0.6585 \times 10^{6}$ and $S_{\text {rated }}$ is the rated power of the cable.

\section{OPTIMIZATION METHOD}

A real offshore wind farm may has hundreds of wind turbines, which are geographically spread-out in the range of several to tens kilometers, there are many feasible schemes for the collector system. In this paper a new optimization approach for searching the optimal collector design is presented. It is based on the multiple which is modified to solve the problem of the design a local WT collector, considering multiple radial feeder connected to the PCC, it is the called Open-Multiple Traveling Salesmen Problem (fsomTSP). Furthermore, GA is used to solve the new approach for problem model presented in this paper. A special gene coding developed for this specific formulation is presented in this section too.

\section{A. General Description of the mTSP}

The mTSP can in general be defined as follows: Given a set of $n$ nodes (cities), let there be $m$ salesmen located at a single node. The remaining nodes that are to be visited are called intermediate nodes. In this paper the TSP is modified into the so called Fixed-Start and Open-Multiple Traveling Salesmen Problem (fsomTSP). This consists of finding tours for all $m$ salesmen, such that all salesman starts its own travel from one fixed-location city, travels to a unique set of cities (none of them close their loops) without returning to the starting location. Except for the first city (common starting point), each city is visited by exactly one salesman.

The mathematical definition of the problem starts from graph theory. Let graph $G=(V, E)$, a weighted undirected graph with cost matrix $C$, where $V$ is the set of $n$ nodes (vertices) $\{1,2, . . n\}$, and $E$ is the set of arcs (edges). The fsomTSP consists of finding a set of sub-graphs $G_{1}\left(V_{1}, E_{1}\right)$, $G_{2}\left(V_{2}, E_{2}\right), \ldots, G_{M}\left(V_{m}, E_{m}\right)$, such that satisfy:

$$
\begin{array}{ll}
V_{i} \bigcap_{i \neq j} V_{j}=v_{1} & i, j=1,2, \ldots, m \\
E_{i} \bigcap_{i \neq j} E_{j}=\varnothing & i, j=1,2, \ldots, m
\end{array}
$$

Cost on edge $e=\left\{v_{i}, v_{j}\right\}$ are $c(e)=c_{i j}=c_{j i}$. All costs are positive. The cost metric can be defined in terms of distance, time, etc. Although the TSP has received a great deal of attention, the research on the mTSP is limited [6], [7] and the fsomTSP the lack of research is larger.

\section{B. MTSP model for the local WT collector design Problem [7]}

To convert the fsomTSP into the problem of collector design, the $n$ nodes (vertices) and $m$ salesman may be viewed as $N_{W T}$ wind turbines plus the PCC $\left(n=N_{W T}+1\right)$ and $m$ feeders in the local WT collector system. Let $\mathbf{F}_{\pi}=\left\{F_{\pi(1)}, F_{\pi(2)}\right.$, $\left.\ldots, F_{\pi(\mathrm{m})}\right\}$ a set of all feeders $F_{\pi(\mathrm{i})}$, where all vertices are visited exactly once (one per WT) and the starting point in common $\left(v_{l}\right)$.

$$
\begin{gathered}
\mathbf{F}_{\pi}=F_{\pi(1)}, F_{\pi(2)}, \ldots F_{\pi(m-1)}, F_{\pi(m)} \\
F_{\pi(i)} \bigcap_{\forall i, j} F_{\pi(j)}=v_{1} \\
F_{\pi(i)} \bigcup_{\forall i, j} F_{\pi(j)}=V
\end{gathered}
$$

The cost of the feeder $C\left(F_{\pi(i)}\right)$ is the sum of the cost of the conductor size used to walk the feeder $C_{\pi}\left(e_{\pi(i)}\right)$.

$$
C\left(F_{\pi(i)}\right)=\sum_{j \in V_{\pi(i)}} C_{\pi}\left(e_{\pi(i)}\right)
$$

The cost of this collector system $C\left(F_{\pi}\right)$ is the sum of the cost of the each feeder:

$$
C\left(F_{\pi}\right)=\sum_{j \in F_{\pi}}^{m} C\left(F_{\pi(i)}\right)
$$

The output is the set of feeders $F$ together its cost $C(F)$ in the feeder of minimum cost, $O P T$. 


$$
\forall F_{\pi}: O P T=C(F) \leq C\left(F_{\pi}\right)
$$

Note that this set of feeders is not necessary unique.

\section{Genetic Algorithm for the fsoTSP}

There are many possible configurations for the local WT collector system of one offshore wind farm; it is a problem of combinatorial optimization and is suitable for intelligent heuristic methods. GA has demonstrated special efficacy when searching and optimizing problems that have huge solution spaces with discrete variables; the optimal design of an offshore wind farm layout is such a problem. The efficiency of the GA can be improved for the specific case of this problem based on: consider radial topology on the local WT collector system and GA is combined with the solution approach used to solve the classical fsomTSP. The main input data of the optimization problem is the coordinates $\left(x_{i}, y_{j}\right)$ of the $N_{W T}$ WT and the transformer substation (for simplicity $\left(x_{1}, y_{1}\right)$ is the location of the PCC), which is determined by micro-sitting assessment, this is then not part of the optimization problem. The flowchart of the optimization approach is show in Fig. 3, and it is drawn under the following general subsections.

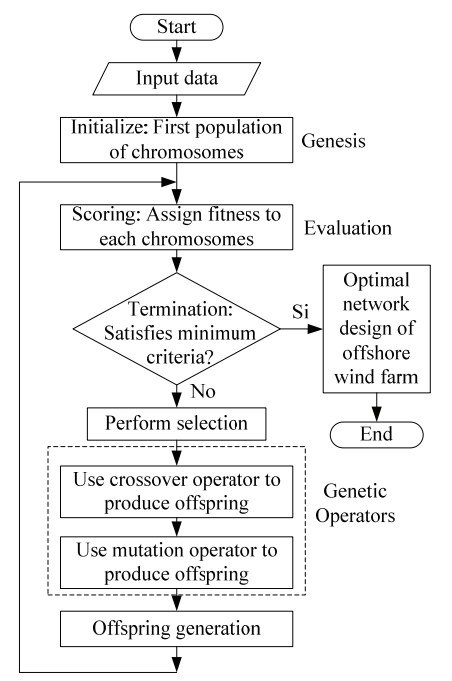

Fig. 3. Flowchart of the optimization approach.

1) Variables and Chromosome: Encoding

Coding all potential solutions to the chromosomes is the first step in solving the optimization problem [8], [9]; in this case one chromosome represents an set of feeder in the local WT collector system and its characteristics (Fig. 4).

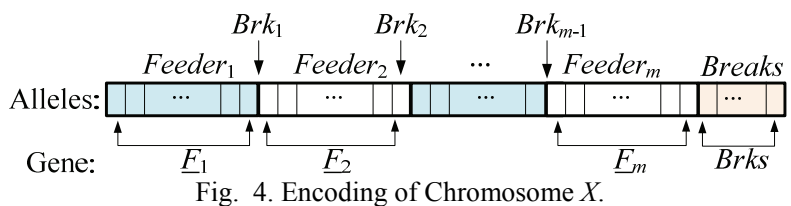

The chromosome $X$ contain $m+1$ genes: genes $\underline{F}_{i},(i=1$, $\ldots, m)$, and one called Brks. The $\underline{F}_{i}$ genes represent one simplification the Feeder $_{i}\left(F_{i}\right)$. It represents the description of the connection topology between the WTs in the feeder $i$ th to and PCC, but where the PCC has been deleted in the allele representation because it is common to all feeders and it is defined as 1 . Breaks contain the alleles which locus for the $m$ 1 breakpoint $\left(B r k_{i}\right)$ inside the whole chromosome. The coding used for the feeders topology is illustrated with an example (Fig. 5) which consist of $N_{w t}=16 \times 2 \mathrm{MW}$ wind turbines connected to one substation.

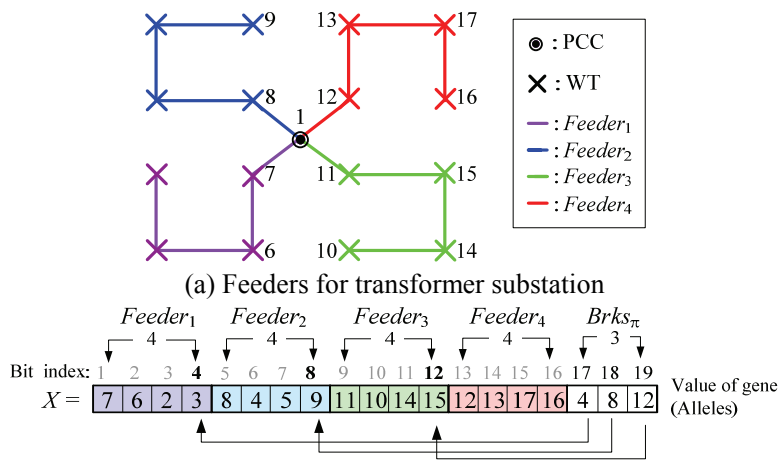

(b) Coding of gene $X$

Fig. 5. Example of the coding for transformer substation and $X$.

Four feeders $(m=4)$ are used on the offshore transformer substation to collect the power produced by WTs, then $n=17$. Equal number of wind turbines $\left(N_{W T} / m=4\right)$ has been assumed in each feeder. A possible feeder/break combination might be:

$$
\begin{gathered}
F_{\pi}=\{7,6,2,3,8,4,5,9,11,10,14,15,12,13,17,16\} \\
\text { Brks }_{\pi}=\{4,8,12\}
\end{gathered}
$$

where the point 1 represent the $\mathrm{PCC}$ in the transformer substation and 17 represent the location of the 16th WT. Fig. 5(a) show the topology of the local WT collector system, the 4 feeders connected to the transformer substation. The power of the 4 WTs is clustered per each feeder, the collector system has been optimized in terms of: (a) the cables sizes to satisfy: load current and minimize the voltage drop $\left(\Delta V_{\text {min }}\right)$ throughout the feeder, and (b) the pattern of connection between wind turbines has been optimized in order to minimize distances between them and the PCC.

The gene $X$ contain the information (alleles) regarding of the four wind turbines collected by each feeder Feeder 1 , Feeder $_{2}$, Feeder $_{3}$ and Feeder 4 , and the breakpoints inside the gene $(B r k s)$ in order to define the connectivity in the radial topology for every feeder, see Fig. 5(b). The Feeder ${ }_{1}$ start from substation (point 1) and it connects the WTs numbered as 2, 3, 6 and 7. Brks 1 define the position inside of the gene (locus) where the feeders start and end, Feeder ${ }_{1}$ ends at the locus 4, while Feeders 2 and Feeder 3 end at locus 8 and 12 respectively.

\section{2) Initialization: Genesis}

The origin of the evolution process is generating one random population of $N_{\text {pop }}$ chromosomes which are suitable solutions for the problem. The first generation of genes for the set of feeders topology are created from random permutations of the numbered WT for possible $\underline{F} /$ Brks sequences. 


\section{3) Scoring: Fitness}

During the evolution process the population is evaluated for each generation in order to select chromosome from the existing population and breed a new generation. The chromosomes are selected through a fitness-based process, where fitness is measured by a fitness function. The fitness function $(F)$ used is the reciprocal of the whole cost of the wind farm:

$$
F=\frac{1}{C_{\text {Total }}}
$$

The chromosomes that represent solutions of lower cost have higher probability of evolving during this selection process.

\section{Genetic operator}

Genetic operators (GO) are used in GA to: maintain genetic diversity during the process of evolution, encourage the recombination of excellent genes and introduce new chromosomes. There are two GO in the proposed algorithm: crossover and mutation. They are applied separately for feeders and breaks into the genes used to represent feeder topologies. The GO mutation is used to randomly change the gene alleles. The purpose of the mutation GA is to prevent the genetic population from converging to a local minimum and to introduce to the population new possible solutions. The crossover operator is used in order to produce the next generation by combining the fittest individuals. Each pair of selected individuals produces a pair of new individuals for the next generation.

\section{Simulations AND Results}

To demonstrate the capabilities and application of the approach presented in this paper for the optimal offshore wind farms' collector design a numerical experiment is illustrated. Theoretical analysis over hypothetical wind farms is presented. The test is performed considering $2 \mathrm{MW}, D=80 \mathrm{~m}$ diameter wind turbines and the collector system has been designed using XLPE (cross-linked polyethylene) insulated undersea cables rated at $35 \mathrm{kV}$; maximum voltage drop of $2 \%$ allowable is considered during the cable sizing. The optimization problem has been solved considering the life time of the wind farm considered in this work will be 20 years, with an interest rate of $4 \%$ and profit of $3 \%$.

A cluster of machines in a wind farm must be maintained certain spacing between the wind towers in order to optimize the power cropping. In this paper the test wind farms used for numerical simulations has tower spacing of 4-rotor-diameters along its rows and 7-diameter spacing between rows $(4 D \times$ $7 D$ ) as shown in Fig. 6, the number of wind turbines has increased and same time that number of feeders $\left(m=\left(N_{W T}\right)^{0.5}\right)$.

In the following tests, the GA parameters are: population size is $\left(N_{W T}+1\right)^{0.5}$ individuals; the maximum generation is $200\left(N_{W T}+1\right)^{0.5}$ crossover probability is 0.8 , mutation probability is 0.01 . The parameters used for the GA can be optimized; however, in this paper a short demonstration about the application is presented. Table I shows a summary of the results of increase the impact on the results for different cases considering different quantities of WT and feeders. Results shows the collector system design with minimum cost may be the same solution of minimal feeder's length; however it is not necessary true in al cases, especially for wind farms with a large number of WT.

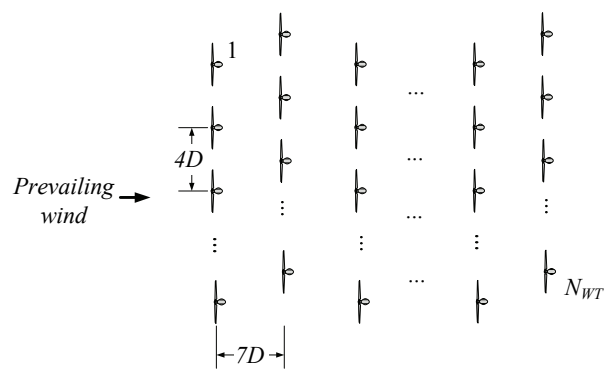

Fig. 6. Wind farm layout considered.

TABLE I. SUMMARY OF RESULTS FOR DIFFERENT HYPOTHETICAL WIND FARMS

\begin{tabular}{cccccc}
\hline \hline Case & $N_{W T}$ & $m$ & $\begin{array}{c}C(F) \\
\text { Total Cost }\end{array}$ & $\begin{array}{c}\text { Total } \\
\text { distance }\end{array}$ & $\begin{array}{c}\text { Minimal } \\
\text { distance }\end{array}$ \\
\hline$I$ & 9 & 3 & 0.64021 & 3374.07 & 3374.07 \\
$I I$ & 16 & 4 & 0.79221 & 6209.60 & 6209.60 \\
$I I I$ & 25 & 5 & 1.53274 & 10114.00 & 10114.00 \\
$I V$ & 36 & 6 & 3.01866 & 15715.84 & 15017.46 \\
$V$ & 49 & 7 & 3.31001 & 21834.39 & 21052.18 \\
$V I$ & 64 & 8 & 3.65047 & 29219.69 & 29219.69 \\
\hline
\end{tabular}

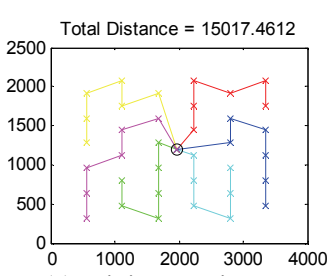

(a) Minimum Distance

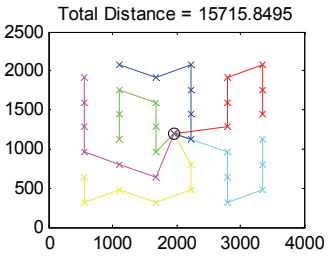

(b) Minimum Cost
Fig. 7. Comparison between the optimal solution of collector system considering (a) minimal distances and (b) minimum cost. Case IV.

Fig. 7 shows the optimization results in terms of cost and distances for the Case $\mathrm{IV}$, it is evident the collector design for minimum cost not coincide with the minimum distance. $t$ is because the increasing of the conductor size in those sections from the end of feeder to the PCC. All the solutions of the optimal design of the collector system for the cases considered are shown in Fig. 8.

The typical convergence curves for all cases tested are shown in Fig. 9. The plots show how the total cost is minimized when the algorithm is progressing through 26 generations in the Case I, instead the largest wind farm, Case $V I$, where over 870 generations the error is less than $5 \%$. It is clear that in each particular case the number of generations could even be reduced without any significant loss of 
accuracy, which means that the estimation time could be also reduced.
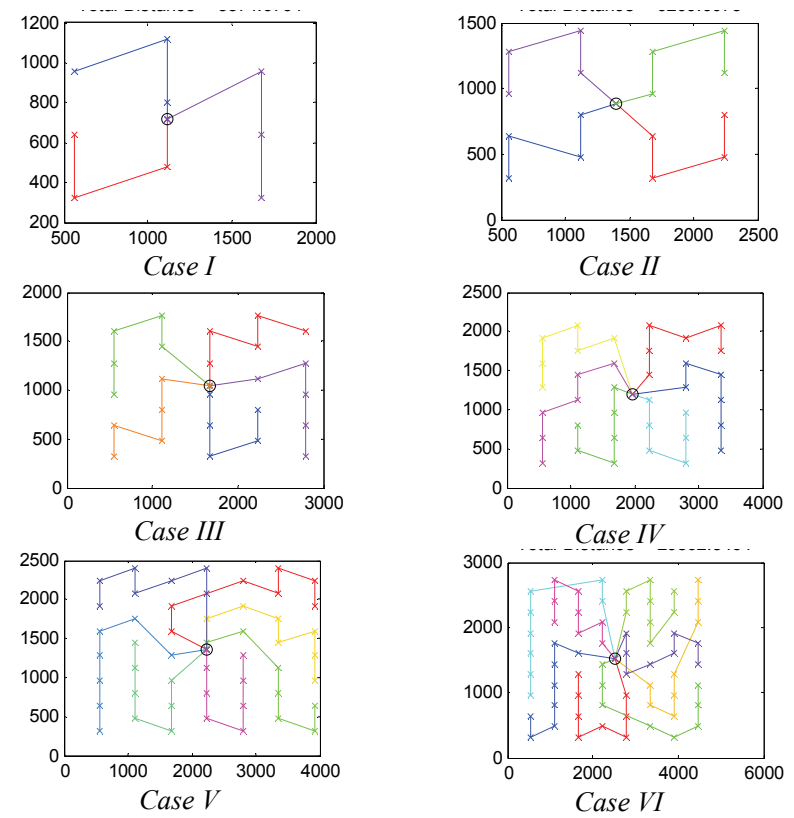

Fig. 8. Wind farm layout considered.

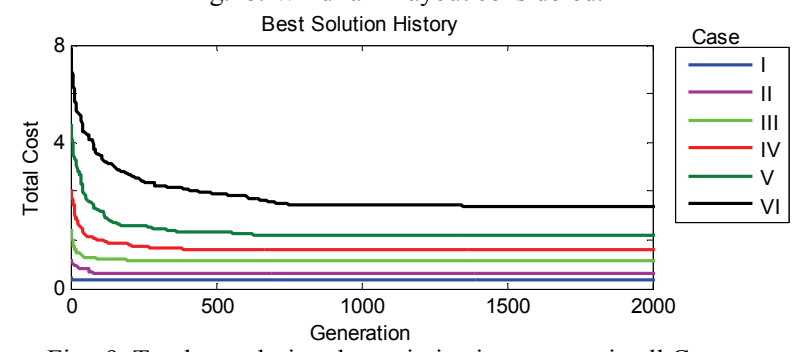

Fig. 9. Total cost during the optimization process in all Cases.

\section{CONCLUSIONS}

A novel approach for solving the problem of optimal electric network design for large offshore wind farms is presented in this paper. It is based on improved GA with the inclusion of a modified version of the multiple traveling salesman problem named Fixed-Start and Open-Multiple Traveling Salesmen Problem.

One special advantage of this approach is that it is fast and effective for radial configuration designs and provides the designer the opportunity to consider different cable cross sections in the design of the local WT collection system.
This offers the opportunity to reduce the cost of the undersea cables used to support the offshore wind farm. Furthermore, an improved cost model has been presented which include more realistic terms for the cost of the transformers connected to WTs and undersea cables. There are several improvements to the optimization model; one of them is to add estimation of power losses on collector system. It is under development at the moment and there are enough discussion results and discussions in this subject for one more paper.

\section{REFERENCES}

[1] EWEA. (2013, 10 January 2013). The European Wind Energy Association homepage | EWEA. Available: http://www.ewea.org

[2] F. M. Gonzalez-Longatt, P. Wall, P. Regulski, and V. Terzija, "Optimal Electric Network Design for a Large Offshore Wind Farm Based on a Modified Genetic Algorithm Approach," Systems Journal, IEEE, vol. 6, pp. 164-172, 2012.

[3] D. D. Li, C. He, and Y. Fu, "Optimization of internal electric connection system of large offshore wind farm with hybrid genetic and immune algorithm," in Electric Utility Deregulation and Restructuring and Power Technologies, 2008. DRPT 2008. Third International Conference on, 2008, pp. 2476-2481.

[4] H. Lingling, F. Yang, and G. Xiaoming, "Optimization of electrical connection scheme for large offshore wind farm with genetic algorithm," in Sustainable Power Generation and Supply, 2009. SUPERGEN '09. International Conference on, 2009, pp. 1-4.

[5] M. Nandigam and S. K. Dhali, "Optimal design of an offshore wind farm layout," in Power Electronics, Electrical Drives, Automation and Motion, 2008. SPEEDAM 2008. International Symposium on, 2008, pp. 1470-1474.

[6] T. Bektas, "The multiple traveling salesman problem: an overview of formulations and solution procedures," Omega, vol. 34, pp. 209-219, 2006.

[7] L. Tang, J. Liu, A. Rong, and Z. Yang, "A multiple traveling salesman problem model for hot rolling scheduling in Shanghai Baoshan Iron \& Steel Complex," European Journal of Operational Research, vol. 124, pp. 267-282, 2000.

[8] M. Gen and R. Cheng, Genetic Algorithms and Engineering Optimization. Canada: John Wiley and Sons, Inc., 2000.

[9] R. L. Haupt and S. E. Haupt, Practical Genetic Algorithms, 2nd ed. Hoboken, New Jersey: John Wiley \& Sons, Inc., 2004. 